\title{
The Value of Bacterial Culture During Clean Orthopedic Surgery: A Prospective Study of 1,036 Patients
}

Louis Bernard, MD; Christophe Sadowski, MD; Daniel Monin, MD; Richard Stern, MD; Blaise Wyssa, MD; Peter Rohner, PhD; Daniel Lew, MD; Pierre Hoffmeyer, MD; Groupe d'Etude sur l'Ostéite

\begin{abstract}
OBJECTIVE: To determine whether bacterial cultures of the wounds of patients undergoing clean orthopedic surgery would help predict infection.

METHODS: During 1 year, 1,256 cultures were performed for 1,102 patients who underwent clean orthopedic surgery. Results were analyzed to evaluate their ability to predict postoperative infection.

RESULTS: The sensitivity, specificity, positive predictive value, and negative predictive value of the cultures were $38 \%$, $92 \%, 7 \%$, and $99 \%$, respectively.

CONCLUSIONS: Cultures performed during clean orthopedic surgery were not useful for predicting postoperative infection (Infect Control Hosp Epidemiol 2004;25:512-514).
\end{abstract}

Despite advances in operative technique, better understanding of the pathogenesis of wound infections, and the widely accepted use of prophylactic antibiotics, postoperative infection continues to be a major source of morbidity and mortality for patients undergoing surgical procedures. In orthopedic surgery, isolation of microorganisms from bone or joint culture is the standard for determining whether a bacterial infection is present. In clean or thopedic surgery, it can be difficult to determine whether organisms isolated from the wound during surgery are pathogenic or simply contaminants. ${ }^{1 \cdot 3} \mathrm{~A}$ wound culture performed during surgery is relatively expensive (\$25 U.S. per culture).

We conducted a prospective study of deep wound cultures performed during clean orthopedic surgery to evaluate their efficacy for predicting postoperative infection.

\section{METHODS}

\section{Patients}

All patients undergoing clean orthopedic surgery in our hospital from December 1, 1997, to December 1, 1998, were consecutively enrolled in the study. Patients without follow-up were excluded from this study.

For patients undergoing surgery without the use of an implant, the length of follow-up was 1 month. For procedures performed with the use of an implant (prosthesis or fracture fixation device), patients were observed for 1 year after surgery. Patients with an implant and a positive intraoperative wound culture were observed for 2 additional years. At each follow-up visit, patients were evaluated for infection.

\section{Wound Culture}

At the end of the surgical procedure, just prior to fascial closure, a sterile cotton swab was applied to the implant, bone, or joint surface. There was no standard technique for obtaining specimens for culture. It was left to the operating surgeon to sample the surgical field. This likely varied from surgeon to surgeon and there was no direct observation of surgeons' sampling technique. Swabs were immediately transported to the bacteriology laboratory in modified Amies transport medium.

\section{Microbiological Study}

Under sterile conditions, swabs were used to inoculate brain-heart infusion broth using standard methods (aerobic and anaerobic) before transfer to plates, which were incubated at $37^{\circ} \mathrm{C}$ and examined every day for 7 days. Bacteria were isolated using brain-heart infusion agar, identified with the API Identification System (bioMérieux Diagnostics, Marcy l'Etoile, France), and subjected to susceptibility testing. Wound cultures were considered negative if both aerobic and anaerobic broth cultures were negative. A single positive culture qualified

Drs. Bernard, Sadowski, Monin, Stern, Wyssa, and Hoffmeyer are from the Orthopaedic Clinic; Drs. Bernard, Rohner, and Lew are from the Department of Microbiology; and Drs. Bernard and Lew are from the Division of Infectious Diseases, Geneva University Hospital, Geneva, Switzerland. Dr. Bernard is also from the Division of Infectious Diseases, Raymond Poincaré University Hospital, Garches, France.

Address reprint requests to Dr. Louis Bernard, Division of Infectious Diseases, Geneva University Hospital, 24 rue Micheli-du-Crest, 1211 Geneva 14, Switzerland.

The authors thank the surgeons of the orthopedic clinic for inclusion of their patients in this study. 
TABLE 1

Microorganisms Obtained From the Positive INTRAOPERATIVE CULTURES

\begin{tabular}{lc}
\hline Bacteria & No. (\%) \\
\hline Coagulase-negative staphylococci & $51(53)$ \\
Propionibacterium species & $22(23)$ \\
Corynebacterium species & $11(11)$ \\
Streptococcus species & $4(4)$ \\
Bacillus species & $4(4)$ \\
Micrococcus species & $1(1)$ \\
Proteus mirabilis & $1(1)$ \\
Escherichia coli & $1(1)$ \\
Stomatococcus mucilaginous & $1(1)$ \\
Streptococcus species and & $1(1)$ \\
Propionibacterium species & \\
Total & $97(100)$ \\
\hline
\end{tabular}

a patient as having a positive deep wound culture. The physicians were not blinded to the culture results of the study, but no preemptive antibiotic therapy was given to patients with positive intraoperative cultures.

\section{Definition of Infection}

Postoperative infection was defined as infection of the surgical site within 30 days after the operation if there was no implant present, or within 1 year if there was an implant. Superficial infection was defined as infection involving only the skin or subcutaneous tissue (superficial to the fascia), and deep infection was defined as that involving the tissues deep to the fascial plane. ${ }^{4} \mathrm{~A}$ clinical examination was performed on all patients included in the study. For patients with abnormal findings on physical examination, laboratory tests, plain radiographs, aspiration, ultrasonography, and radionucleotide imaging were used to confirm a diagnosis of infection.

\section{Statistical Analysis}

Data were prospectively analyzed using a specially designed case report form, with one form per patient. The following data were recorded onto an electronic spreadsheet (Microsoft Excel, Microsoft Corp., Redmond, WA): (1) date and type of operation; (2) date and number of positive cultures; (3) date of diagnosis of any postoperative infection; and (4) the type of microorganism isolated. The sensitivity, specificity, positive predictive value, and negative predictive value of the intraoperative wound cultures for predicting infection were calculated using the number of individual operations as the denominator, not the number of patients. Statistical analysis was performed using Statview 5 software (SAS Institute, Inc., Cary, NC). Continuous variables were presented as mean and standard deviation, and categorical variables as number and percentage. The results were presented according to a subgroup definition, patients with and without positive cultures at the time of surgery.
TABLE 2

Literature ReVIEW of The Value of INTRaOperative Cultures in Clean Orthopedic Surgery

\begin{tabular}{|c|c|c|c|}
\hline Study & $\begin{array}{l}\text { No. of } \\
\text { Patients or } \\
\text { Wounds }\end{array}$ & $\begin{array}{r}\text { Co } \\
\text { E } \\
\text { I }\end{array}$ & $\begin{array}{l}\text { Correlation } \\
\text { Between } \\
\text { Positive } \\
\text { Culture and } \\
\text { Risk of } \\
\text { Infection }\end{array}$ \\
\hline Lindgren et al. ${ }^{1}$ & 107 & Skin, drain tip & Yes \\
\hline Dietz et al. ${ }^{2}$ & 40 & Tissue, fluids & No \\
\hline Moussa et al. ${ }^{3}$ & 21 & Wound, fluids & No \\
\hline Lindahl et al. ${ }^{7}$ & 60 & Synovial, suction drainage & Yes \\
\hline Overgaard et al. ${ }^{8}$ & 78 & Wound, drain tip, drainage & No \\
\hline Harwood et al. ${ }^{9}$ & 425 & Fluids, drain tip & No \\
\hline Fitzgerald et al..$^{10}$ & 658 & Wound, fluids & Yes \\
\hline Dobbins et al. ${ }^{11}$ & 26 & Wound, fluids & Yes \\
\hline Current study & 1,036 & Fluids near implants or bone & No \\
\hline
\end{tabular}

\section{RESULTS \\ Patients}

From the total study population of 1,102 patients, 66 patients were excluded because of lack of follow-up. The final study population thus included 1,036 patients (469 males and 567 females) with a mean age of 52 years (standard deviation, \pm 21 years; range, 15 to 95 years). All patients had received antimicrobial prophylaxis with cefuroxime or vancomycin. Operative procedures included prosthetic joint surgery $(n=507,43 \%)$, open reduction internal fixation of fractures $(n=472,40 \%)$, and others (eg, osteotomy, hardware removal, and spine surgery; $n=201,17 \%$ ).

\section{Deep Wound Cultures}

Sixteen patients developed an infection following 1,180 clean orthopedic operations (1,180 cultures among 1,036 patients). Causative microbes included methicillinresistant Staphylococcus aureus $(\mathrm{n}=6)$, methicillin-sensitive $S$. aureus $(\mathrm{n}=3)$, coagulase-negative staphylococci $(\mathrm{n}=2)$, Pseudomonas aeruginosa $(\mathrm{n}=2)$, Enterococcus faecalis $(\mathrm{n}=2)$, and Enterobacter cloacae $(\mathrm{n}=2)$. Most of the infecting pathogens $(81 \%)$ were resistant to the drug chosen for prophylaxis. There were a total of 1,083 negative cultures $(99 \%) ; 10$ of these patients developed a postoperative infection ( 3 superficial and 7 deep). Positive cultures were noted after 97 operations (8.3\%); 6 were followed by an infection (4 superficial and 2 deep) (relative risk, 6.7; $95 \%$ confidence interval, 2.49 to $18.04 ; P=.001$ ). Thus, in these clean orthopedic procedures, the sensitivity, specificity, positive predictive value, and negative predictive value of a deep wound culture to detect a postoperative infection were $38 \%, 92 \%, 7 \%$, and $99 \%$, respectively.

\section{Bacterial Identification}

The most frequently identified bacterial organisms were coagulase-negative staphylococci (53\%), Propioni- 
bacterium species (23\%), and Corynebacterium species (11\%). In all instances in which these organisms were isolated from the intraoperative wound culture, no postoperative infection developed due to microbes isolated. Moreover, the bacteria growing in intraoperative wound cultures of patients who developed an infection $(n=6)$ were different from those responsible for the postoperative infection. The microorganisms obtained from the intraoperative wound cultures are listed in Table 1.

\section{DISCUSSION}

Most of the patients $(62 \%)$ who developed an infection following clean orthopedic surgery had negative deep wound intraoperative cultures. Three-quarters of the bacteria isolated from positive cultures were coagulase-negative staphylococci and Propionibacterium species (74\%), and these cases did not have postoperative infection. Coagulase-negative staphylococci and Propionibacterium have been frequently associated with late infected prostheses. ${ }^{5,6}$ However, none of our patients with an implant and an intraoperative wound culture positive for these organisms showed any evidence of infection after 3 years of follow-up. Additionally, the 6 patients who developed an infection after having a positive intraoperative culture did so with a different organism. Therefore, none of the 97 patients with a positive culture became infected with the organism cultured at the time of their surgery.

The lack of bacterial growth does not necessarily imply that the surgical field was sterile. A sterile surgical field could explain the development of a postoperative infection in 10 patients who had negative intraoperative cultures, or some of these patients may have acquired a new infection postoperatively. It is problematic that patients developed a postoperative infection with an organism different from that which was cultured in the operating room, and we do not have a satisfactory explanation for this. It is well known that in clean orthopedic surgery most, if not all, patients receive some antibiotic perioperatively as prophylaxis. In our institution, either cefuroxime or vancomycin is used in this regard. This practice did not change during the period of our study and we did not correlate our results with the specific drug being used.

Eight studies have evaluated the use of intraoperative deep wound cultures, cultures of wound suction drainage, or both for predicting infection following clean orthopedic surgery (Table 2) ${ }^{1-3,7-11}$ Half of the studies have reported that the results of such cultures predict infection. Most of the prior studies of this question have used antibiotic prophylaxis, so this variable does not appear to explain the difference between positive and negative studies. In a recent study, we found that a positive suction drainage culture did not predict postoperative infection for clean or thopedic surgery, but was highly predictive of persistent sepsis for septic or thopedic surgery. ${ }^{12}$ Although the current study found that intraoperative deep wound cultures did not predict infection following clean surgery, it is possible that the risk of infection would be higher in patients with virulent bacteria (eg, S. aureus). It was of interest that patients with a positive intraoperative culture were 6.7-fold more likely to develop postoperative infection than were those with a negative intraoperative culture. This may be because the infecting microbe was present in lower (undetectable) numbers, but was more virulent than the one that was detected.

\section{REFERENCES}

1. Lindgren U, Elmros T, Holm SE. Bacteria in hip surgery: a study of routine aerobic and anaerobic cultivation from skin and closed suction wound drains. Acta Orthop Scand 1976;47:320-323.

2. Dietz FR, Koontz FP, Found EM, Marsh JL. The importance of positive bacterial cultures of specimens obtained during clean orthopaedic operations. J Bone Joint Surg Am 1991;73:1200-1207.

3. Moussa FW, Anglen JO, Gehrke JC, Christensen G, Simpson WA. The significance of positive cultures from orthopaedic fixation devices in the absence of clinical infection. Am J Orthop 1997;26:617-620.

4. Wong ES. Surgical site infections. In: Mayhall G, ed. Hospital Epidemiology and Infection Control, ed. 2. Philadelphia: Lippincott Williams \& Wilkins; 1999:204.

5. Vandecasteele SJ, Van Wijngaerden E, Van Eldere J, Peetermans WE. New insights in the pathogenesis of foreign body infections with coagulase negative staphylococci. Acta Clin Belg 2000;55:148-153.

6. Tunney MM, Patrick S, Curran MD, et al. Detection of prosthetic hip infection at revision arthroplasty by immunofluorescence microscopy and PCR amplification of the bacterial $16 \mathrm{~S}$ rRNA gene. J Clin Microbiol 1999;37:3281-3290.

7. Lindahl J, Korkala O, Pammo H, Miettinen A. Bacterial contamination and closed suction drainage in open meniscectomy of the knee. Ann Chir Gynaecol 1993;82:51-54.

8. Overgaard S, Thomsen NO, Kulinski B, Mossing NB. Closed suction drainage after hip arthroplasty: prospective study of bacterial contamination in 81 cases. Acta Orthop Scand 1993;64:417-420.

9. Harwood DA, Robbins SG, Zawadsky JP, Bullek D. The predictive value of intraoperative and post-operative cultures in patients undergoing total hip arthroplasty. Orthopaedic Transactions 1989;13:60-61.

10. Fitzgerald RH Jr, Peterson LF, Washington JA 2nd, Van Scoy RE, Coventry MB. Bacterial colonization of wounds and sepsis in total hip arthroplasty. J Bone Joint Surg Am 1973;55:1242-1250.

11. Dobbins JJ, Seligson D, Raff MJ. Bacterial colonization of or thopaedic fixation devices in the absence of clinical infection. $J$ Infect $D$ is 1988;158:203-205.

12. Bernard L, Pron B, Vuagnat A, et al. The value of suction drainage culture during clean and septic orthopaedic operations: a prospective study of 901 patients. Clin Infect Dis 2002;34:46-49. 